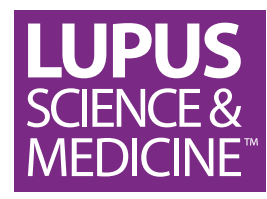

\title{
Association of COVID-19 pandemic- related concern and health routine changes with functioning among individuals with systemic lupus erythematosus
}

\author{
Laura C Plantinga (D) , ${ }^{1,2}$ Courtney Hoge, ${ }^{1}$ Charmayne Dunlop-Thomas, \\ Brad D Pearce, ${ }^{2}$ S Sam Lim (D) , , ${ }^{1,2}$ Cristina Drenkard (D) , ${ }^{1,2}$ C Barrett Bowling ${ }^{3,4}$
}

To cite: Plantinga LC, Hoge C, Dunlop-Thomas C, et al. Association of COVID-19 pandemic-related concern and health routine changes with functioning among individuals with systemic lupus erythematosus. Lupus Science \& Medicine 2022;9:e000658. doi:10.1136/ lupus-2022-000658

Received 4 January 2022 Accepted 11 February 2022

Check for updates

(c) Author(s) (or their employer(s)) 2022. Re-use permitted under CC BY-NC. No commercial re-use. See rights and permissions. Published by BMJ.

${ }^{1}$ Department of Medicine, Emory University, Atlanta, Georgia, USA ${ }^{2}$ Department of Epidemiology, Emory University, Atlanta, Georgia, USA

${ }^{3}$ Durham Veterans Affairs Geriatric Research Education and Clinical Center, Durham Veterans Affairs Medical Center, Durham, North Carolina, USA

${ }^{4}$ Department of Medicine,

Duke University, Durham, North Carolina, USA

Correspondence to Dr Laura C Plantinga; laura. plantinga@emory.edu

\section{ABSTRACT}

Objective To examine whether pandemic-related issues were associated with physical functioning, community mobility and cognition among individuals with SLE.

Methods Participants were recruited (6 October 2020-11 November 2021) for this cross-sectional study from a population-based cohort of individuals with validated SLE in metropolitan Atlanta, as part of an ongoing ancillary study. Pandemic-related issues (concern about the pandemic (very vs somewhat/not at all concerned); changes in physical activity and sleep (less vs more/same); difficulty obtaining food and medications and accessing routine care (any vs none)) were self-reported. Self-reported physical functioning and episodic and working memory performance were reported as t-scores (such that a score of $50=$ population mean and a 10-point difference $=1 \mathrm{SD}$ ) and community mobility scores ranged from 0 to 120, with higher scores representing better functioning for all domains. Differences in scores were assessed via t-tests and age-adjusted, sex-adjusted and raceadjusted linear regression.

Results Among 245 participants (mean age, 46 years; 95\% female, $77 \%$ black), physical functioning t-scores (mean=44) were consistently lower (by 3-5 points) for those who reported concern about the pandemic, less physical activity and sleep, difficulty obtaining food and medications, and accessing routine care. Similarly, community mobility scores (mean=48) were lower (by 10-20 points) for these individuals. There were no substantial differences in episodic memory and working memory t-scores (mean $=50$ and 47 , respectively) by pandemic-related issues.

Conclusion We found that physical functioning and community mobility, but not cognition, were lower among those who reported more concern about the pandemic or greater disruptions to health routines. Future studies should explore interventions among these vulnerable individuals with SLE, who already disproportionately suffer from functional impairment, to maintain functioning and prevent adverse outcomes during times of crisis.

\section{INTRODUCTION}

Individuals with SLE have suboptimal levels of functioning across multiple domains,

\section{Key messages}

What is already known about this subject?

- The COVID-19 pandemic may have adversely affected functioning in individuals with SLE.

- These relatively young individuals are already disproportionately affected by functional impairment.

What does this study add?

- We found that, in a cohort of individuals with SLE, physical functioning and community mobility, but not cognition, were lower among those who reported more concern about the pandemic or greater disruptions to health routines during the pandemic.

How might this impact on clinical practice or future developments?

- Future studies should explore interventions to maintain functioning in this vulnerable population during times of crisis.

including perceived physical functioning, community mobility and social participation, and cognition. ${ }^{1}$ The COVID-19 pandemic has generally been associated with disrupted health routines and fewer opportunities for social, mental and physical activity; fear about COVID-19 may have further limited these opportunities. Thus, concern about the pandemic and pandemic-related changes in health routines, including those directly related to disease self-management, may have negatively impacted daily functioning among these individuals, who are already disproportionately functionally impaired. Leveraging an ongoing ancillary cohort study (Approaches to Positive, Patient-centered Experiences of Aging in Lupus (APPEAL)), we examined whether pandemic-related issues were associated with self-reported physical functioning, 
Table 1 Characteristics of participants with SLE completing a functional assessment and COVID-19 pandemic questionnaire (6 October 2020-11 November 2021)

\begin{tabular}{lc}
\hline Characteristic & Mean (SD) or $\mathbf{n}(\%)$ \\
\hline $\mathrm{N}$ & 245 \\
\hline Demographic & \\
\hline $\begin{array}{l}\text { Mean (SD) age at survey, years } \\
\text { Female at birth, } \mathrm{n}(\%)\end{array}$ & $46.0(11.5)$ \\
\hline Race, ${ }^{*} \mathrm{n}(\%)$ & $233(95.1)$ \\
\hline Black & $193(78.8)$ \\
\hline White & $37(15.1)$ \\
\hline Asian & $4(1.6)$ \\
\hline American Indian/Alaskan Native & $1(0.4)$ \\
\hline Hawaiian/Pacific Islander & $1(0.4)$ \\
\hline Other & $9(3.7)$
\end{tabular}

\section{Pandemic related}

\begin{tabular}{lr} 
Very vs somewhat/not all concerned $†$ & $137(56.2)$ \\
\hline Less physically active $\neq$ & $133(54.7)$ \\
\hline Sleeping less $\ddagger$ & $132(53.9)$ \\
\hline Difficulty obtaining food $\neq$ & $83(33.9)$ \\
\hline Difficulty accessing routine medical care $\neq$ & $103(42.0)$ \\
\hline Difficulty obtaining medications $\ddagger$ & $52(21.2)$ \\
\hline Difficulty obtaining hydroxychloroquine $£ \S$ & $46(29.1)$ \\
\hline
\end{tabular}

*Participants who reported multiple races including black $(n=3)$ were categorised as black; no other participants listed multiple races. $\dagger \mathrm{N}=244$.

$\ddagger$ Reported as less versus about the same or more (physical activity, sleep) or any versus no difficulty (obtaining food, accessing routine care, obtaining medications), as compared with prior to the pandemic. $\S$ Among those who reported currently taking hydroxychloroquine $(\mathrm{N}=158)$.

community mobility and cognitive performance among individuals with SLE.

\section{METHODS}

\section{Study population and data sources}

We recruited patients and used data from the ongoing, population-based Georgians Organized Against Lupus (GOAL) cohort. GOAL recruitment and data collection details have been described ${ }^{2}$; participants are adults $(\geq 18$ years) with a documented diagnosis of SLE ( $\geq 4$ revised American College of Rheumatology (ACR) criteria, ${ }^{3}$ or 3 ACR criteria plus a diagnosis of SLE by an attending board-certified rheumatologist). Participants were ineligible for APPEAL remote visits if they currently lived outside of Georgia, were unable to complete the consent, did not speak English, had insufficient vision or hearing, or did not have a laptop or smartphone or internet access. APPEAL began enrolment on 8 October 2019. In-person visits were temporarily halted (10 March 2020-20 July 2021) at the start of the pandemic and a remote version of the APPEAL Study visit was created, pilot-tested and rolled out. Here, we report on data from remote APPEAL visits (6 October 2020-11 November 2021), which included a COVID-19 questionnaire. Data on cognitive performance were entered using the National Institutes of Health (NIH) Toolbox application ${ }^{45}$; other data were obtained from self-administered surveys via REDCap. ${ }^{6}$

\section{Variables}

Pandemic-related issues

Participants were asked about six potential pandemicrelated issues, ${ }^{7}$ including level of concern about the pandemic (dichotomised as very vs somewhat/not at all concerned) and changes in health routines relative to before the pandemic: level of physical activity and amount of sleep (dichotomised as less vs more/about the same); and difficulties obtaining food, accessing routine medical care, and obtaining medications (dichotomised as very/ unable, much or some vs no difficulty).

\section{Self-reported physical functioning}

The Patient-Reported Outcomes Measurement Information System (PROMIS) Physical Functioning-Short Form $12 \mathrm{a}^{8}$ was used to assess participants' perceptions of their physical functioning. Raw scores were scaled to t-scores $(50=$ average score for a general adult population, $10=1 \mathrm{SD}$ ); higher scores represent better self-reported physical functioning.

\section{Community mobility}

The University of Alabama (UAB) Study of Aging LifeSpace Assessment ${ }^{9}$ was used to capture community mobility and social participation by measuring how far respondents go (from the bedroom to other rooms to outside the home, neighbourhood and town (lifespaces)), as well as how often respondents go to these life-spaces and with how much help over the past 4 weeks. Overall scores range from 0 to 120 ; higher scores representing greater life-space mobility.

\section{Episodic and working memory}

The Picture Sequence Memory Test (in which the participant is asked to recall the order of an increasing number of related pictures presented to them; measures episodic memory) and List Sorting Working Memory Test (in which the participant is asked to verbally recite back lists of related objects presented to them, in size order; measures working memory) were administered remotely ${ }^{10}$ via the NIH Toolbox application. ${ }^{45}$ All individual raw scores were converted to t-scores, which were adjusted for age, sex, race/ethnicity and education; higher scores indicate better cognitive functioning.

\section{Demographics}

Age, sex at birth and race were self-reported during the cognition battery.

\section{Statistical analysis}

Descriptive statistics were used to summarise the participant characteristics and overall functioning scores. Scores were compared by dichotomised responses to pandemic 
issue items using t-test and Wilcoxon rank-sum test. Multivariable linear regression models including age, sex and race, and using complete case analysis were used to obtain adjusted differences and 95\% CIs. All analyses were performed using Stata V.17 (College Station, Texas, USA).

\section{RESULTS}

\section{Participant characteristics}

Participants had a mean age of 46 years and were predominantly female and black (table 1 ). More than half reported being very concerned about the pandemic $(56 \%)$, as well as being less physically active $(55 \%)$ and sleeping less $(54 \%)$, relative to before the pandemic. Difficulties obtaining food $(34 \%)$ and medications $(21 \%)$ and accessing routine medical care $(42 \%)$ were also commonly reported (table 1); 10\%, 22\%, 18\%, 18\%, $16 \%, 9 \%$, and $7 \%$ reported zero, one, two, three, four, five, and all six of these issues, respectively. Among those taking hydroxychloroquine, $29 \%$ reported difficulty obtaining it (table 1).

\section{Associations of functioning outcomes with pandemic-related issues}

Physical functioning t-scores (overall mean $=44$ ) were consistently lower (by $3-5$ points, or $\sim 0.5 \mathrm{SD}$ ) for those who reported having these issues versus not (table 2), and there was a linear trend of lower scores with greater number of pandemic-related issues ( 0 issues: 50 (9); 6 issues: 35 (10); $\mathrm{p}<0.001)$. Differences remained after adjustment for age, sex and race (figure 1). Community mobility scores (overall mean $=48$ ) were lower (by 10-20 points) for these individuals (table 2), and there was a similar trend of lower scores with greater number of pandemic-related issues (0 issues: 63 (32); 6 issues: 27 (23); $\mathrm{p}<0.001)$. Those who reported pandemic-related issues were less likely to report reaching each life-space versus those who did not: for example, participants were less likely to report visiting their neighbourhood at least once in the past month without any assistance if they also reported sleeping less $(52 \%$ vs $76 \%$; $\mathrm{p}<0.001)$ or having difficulty obtaining food ( $54 \%$ vs $67 \%, \mathrm{p}=0.05$ ) or medications ( $44 \%$ vs $68 \%$; $\mathrm{p}=0.002$ ). Rank-sum analyses accounting for the skewed distribution of community mobility scores (median=50, IQR=18-72) and adjustment did not substantially change the associations (figure 1 ). Finally, no substantial differences were seen in the episodic memory and working memory t-scores (overall mean $=50$ and 47 , respectively), regardless of adjustment (table 2, figure 1), and no statistically significant trends by total number of pandemic-related issues were noted. In 2021 vs 2020, physical functioning ( 45 vs 42 ), life-space (48 vs 44 ) and episodic memory (50 vs 48 ) scores were higher overall, but patterns in scores were consistent across years; there was no difference by year in working memory scores or patterns.

\section{DISCUSSION}

We found that concern about the pandemic and reported changes in health routines were common and associated with substantially lower scores for perceived physical functioning and community mobility and social participation, consistent with patterns observed in older adults. ${ }^{11-13}$ In this cross-sectional study, we cannot determine whether these observed associations reflect true causal associations. We also cannot know the direction of the associations, or whether these associations differ from those in the population without SLE. Regardless, these results suggest that lower-functioning patients with SLE may disproportionately lack access to resources, including food, medications and medical care, during times of massive societal shifts like this pandemic, making them more vulnerable to further declines in functioning and poor outcomes.

In contrast, these pandemic-related issues were not associated with lower episodic or working memory scores, despite some evidence in older adults that the pandemic had deleterious effects on cognition. ${ }^{14}$ This lack of association may reflect a true lack of association of these issues with cognition. It is also possible that the cognitive domains of working and episodic memory (the only domains that could be measured remotely via NIH Toolbox ${ }^{10}$ ) are not the domains most immediately affected by the pandemicrelated issues among individuals with SLE. In fact, our pilot showed that episodic and working memory scores were consistent with average scores in the general population; but attention and inhibitory control and processing speed scores were substantially lower $(\sim 1 \mathrm{SD}$ below the average), ${ }^{1}$ suggesting these domains may have been more vulnerable to impairment due to pandemic-related issues among individuals with SLE.

Other limitations deserve mention. Residual confounding, selection bias (introduced through the additional exclusion of individuals unable to do a remote visit) and lack of generalisability are all possible. Additionally, there may have been temporal trends in associations (given rapidly changing policies and availability of resources) that could not be captured with analyses by year alone.

In this cross-sectional study of individuals with SLE, we found that physical functioning and community mobility scores, but not episodic and working memory scores, were lower among those who reported higher concern about the COVID-19 pandemic and greater disruptions to health routines during the pandemic. For this relatively young population that already experiences levels of functional impairment most often associated with older age, further sudden declines in functioning could be devastating to both quantity and quality of life years remaining. Future studies should examine persistence of reduced functioning beyond the pandemic, subsequent physical and mental health outcomes, and patients' perspectives on motivators and barriers to maintaining function in times of crisis. Such data can inform efforts to target the vulnerable individuals with SLE for interventions, 
Table 2 Scores* for physical functioning, community mobility, episodic memory and working memory scores of participants, overall and by pandemic-related issues

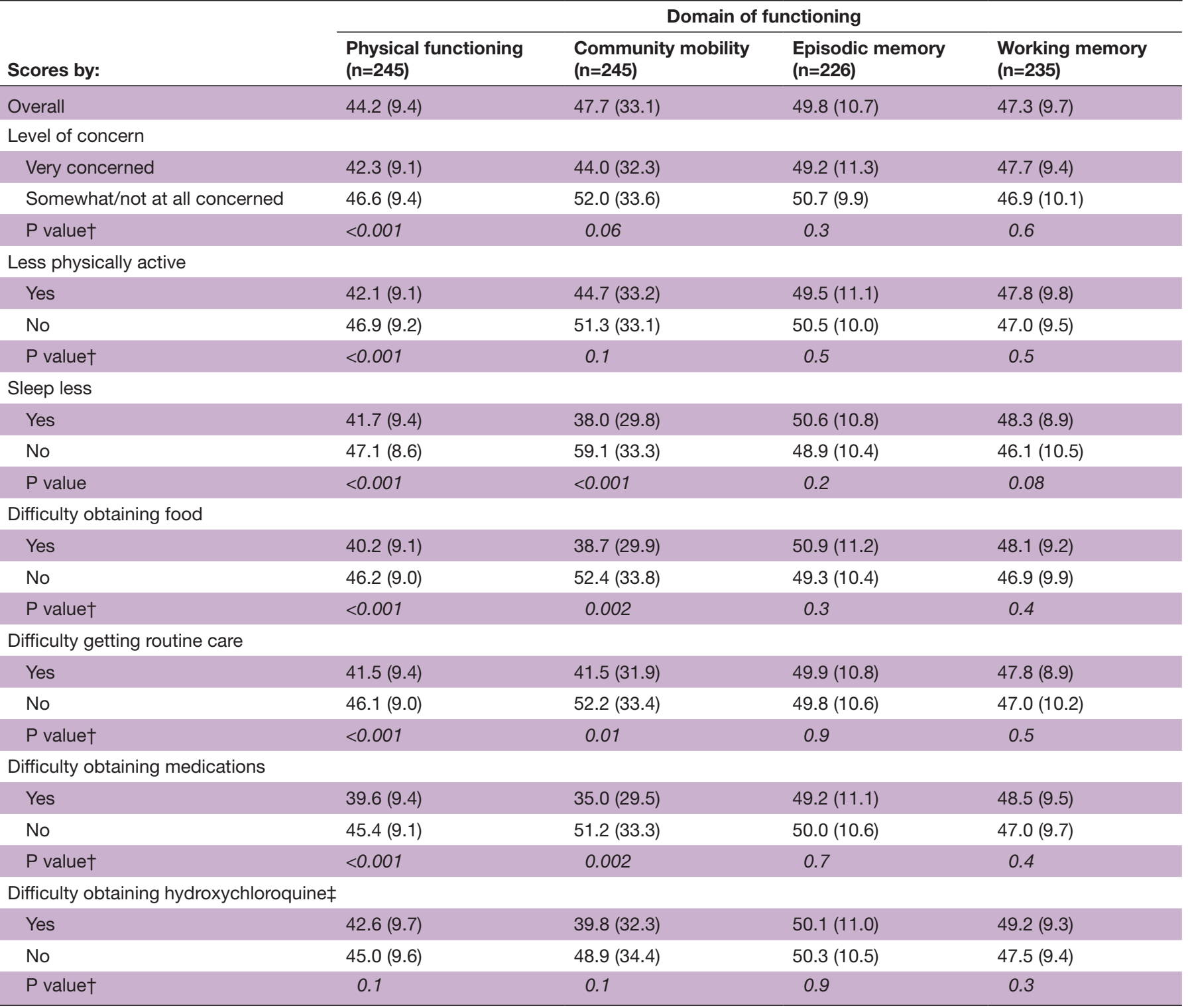

${ }^{*}$ Scores for self-reported physical functioning (from the Patient-Reported Outcomes Measurement Information System Physical Functioning-Short

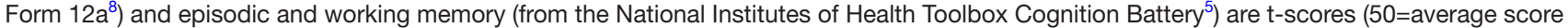
for a general adult population; $10=1 \mathrm{SD}$; and higher scores=better functioning); memory scores are further adjusted for age, sex, race/ethnicity and education. Community mobility scores are from the University of Alabama Study of Aging Life-Space Assessment ${ }^{9}$ (range: 0-120; higher scores $=$ greater community mobility).

†By t-test.

$\ddagger$ Among those who reported currently taking hydroxychloroquine $(n=158)$.

including promotion of physical activity and enhanced social support, to maintain functioning and prevent adverse outcomes during periods of social upheaval.

Acknowledgements We thank the participants of the APPEAL Study, as well as Aita Akharume, Jessie Black and Meaza Girmay for assistance with recruitment and data collection for remote APPEAL visits.

Contributors LP, BDP, SSL, CD and CBB conceptualised and designed the study. LP obtained study funding. $\mathrm{CH}$ and CD-T oversaw recruitment and data collection. $\mathrm{CH}$ piloted the remote study visit and trained the coordinators. All authors provided input to the manuscript and approved the final version. LP is responsible for the overall content as guarantor.
Funding This work was supported by the National Institute on Ageing of the National Institutes of Health (R01AG061179). Support for the GOAL cohort came under the US Centers for Disease Control and Prevention (U01DP006488).

Disclaimer The content is solely the responsibility of the authors and does not necessarily represent the official views of the National Institutes of Health.

Competing interests None declared.

Patient and public involvement Patients and/or the public were not involved in the design, or conduct, or reporting, or dissemination plans of this research.

Patient consent for publication Not required.

Ethics approval This study involves human participants and was approved by Emory Institutional Review Board (IRB00110977). Participants gave informed consent to participate in the study before taking part.

Provenance and peer review Not commissioned; externally peer reviewed. 


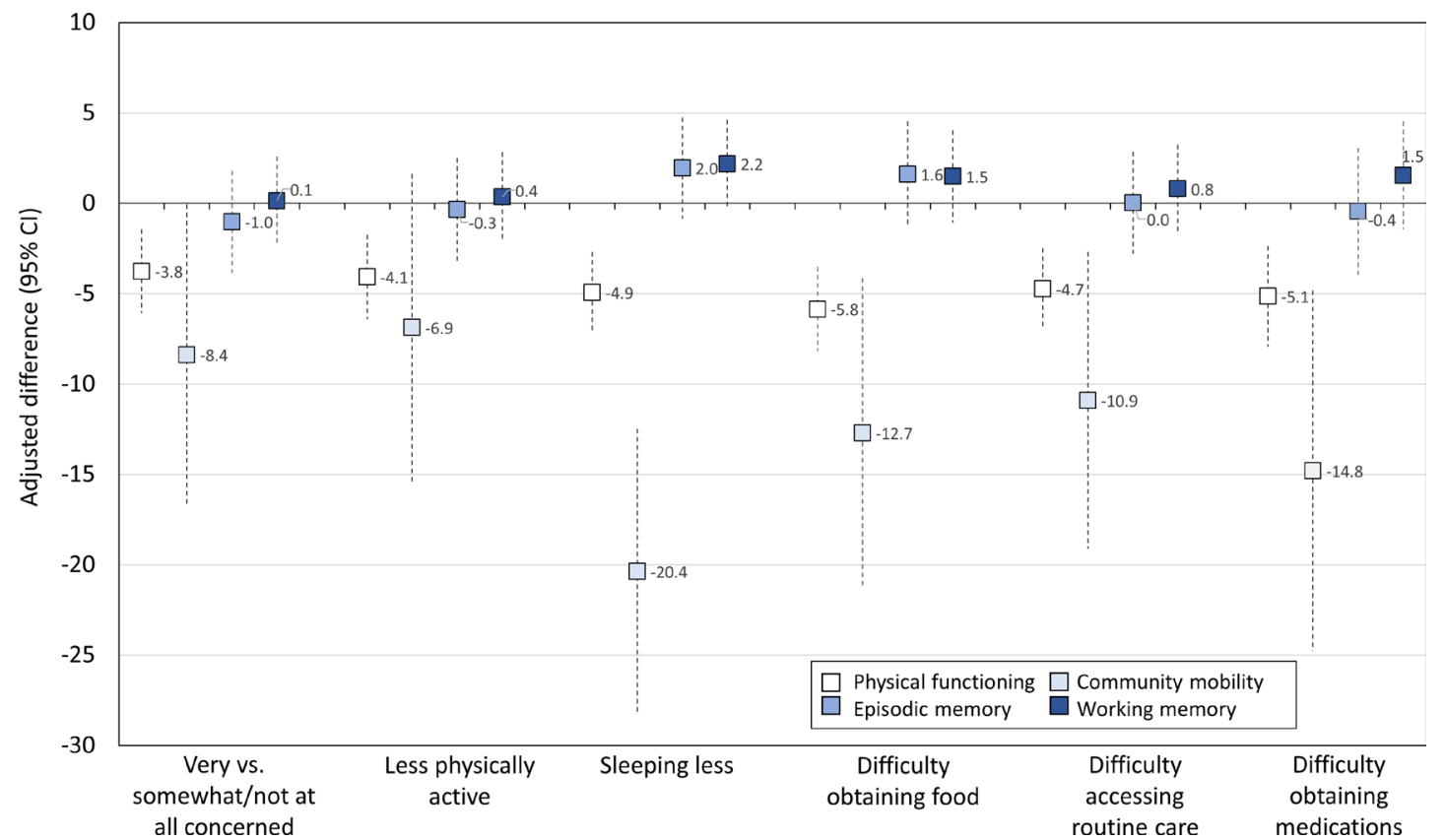

Figure 1 Age-adjusted, sex-adjusted and race-adjusted differences in physical functioning, community mobility, episodic memory and working memory scores by pandemic-related issues. Race was categorised as black versus other. Scores for selfreported physical functioning (from the Patient-Reported Outcomes Measurement Information System Physical FunctioningShort Form $12 \mathrm{a}^{8}$ ) and episodic and working memory (from the National Institutes of Health Toolbox Cognition Battery ${ }^{5}$ ) are t-scores ( $50=$ average score for a general adult population; $10=1 \mathrm{SD}$; and higher scores=better functioning); memory scores are further adjusted for age, sex, race/ethnicity and education. Community mobility scores are from the University of Alabama Study of Aging Life-Space Assessment ${ }^{9}$ (range: 0-120; higher scores=greater community mobility).

Open access This is an open access article distributed in accordance with the Creative Commons Attribution Non Commercial (CC BY-NC 4.0) license, which permits others to distribute, remix, adapt, build upon this work non-commercially, and license their derivative works on different terms, provided the original work is properly cited, appropriate credit is given, any changes made indicated, and the use is non-commercial. See: http://creativecommons.org/licenses/by-nc/4.0/.

\section{ORCID iDs}

Laura C Plantinga http://orcid.org/0000-0003-0809-8981

S Sam Lim http://orcid.org/0000-0003-2361-0787

Cristina Drenkard http://orcid.org/0000-0002-6832-7291

\section{REFERENCES}

1 Plantinga L, Tift BD, Dunlop-Thomas C, et al. Geriatric assessment of physical and cognitive functioning in a diverse cohort of systemic lupus erythematosus patients: a pilot study. Arthritis Care Res 2018;70:1469-77.

2 Lim SS, Drenkard C. Understanding lupus disparities through a social determinants of health framework: the Georgians organized against lupus research cohort. Rheum Dis Clin North Am 2020;46:613-21.

3 Hochberg MC. Updating the American College of rheumatology revised criteria for the classification of systemic lupus erythematosus. Arthritis Rheum 1997;40:1725.

4 Northwestern University. Cognition measures: NIH Toobox cognition batteries, 2017. Available: http://www.healthmeasures.net/exploremeasurement-systems/nih-toolbox/intro-to-nih-toolbox/cognition

5 Weintraub S, Dikmen SS, Heaton RK, et al. The cognition battery of the NIH toolbox for assessment of neurological and behavioral function: validation in an adult sample. J Int Neuropsychol Soc 2014;20:567-78.

6 Harris PA, Taylor R, Thielke R, et al. Research electronic data capture (REDCap)--a metadata-driven methodology and workflow process for providing translational research informatics support. J Biomed Inform 2009;42:377-81.

7 Cawthon PM, Orwoll ES, Ensrud KE, et al. Assessing the impact of the COVID-19 pandemic and accompanying mitigation efforts on older adults. J Gerontol A Biol Sci Med Sci 2020;75:e123-5.

8 Northwestern University. Introduction to PROMIS, 2017. Available: http://www.healthmeasures.net/explore-measurement-systems/ promis/intro-to-promis

9 Peel C, Sawyer Baker P, Roth DL, et al. Assessing mobility in older adults: the UAB study of aging Life-Space assessment. Phys Ther 2005;85:1008-19.

10 National Institutes of Health. Remote adminstration guidelines for the NIH toolbox: response to COVID-19, 2020. Available: https:// nihtoolbox.force.com/s/article/Coronavirus-Covid-19

11 Hoffman GJ, Malani PN, Solway E, et al. Changes in activity levels, physical functioning, and fall risk during the COVID-19 pandemic. J Am Geriatr Soc 2022;70:49-59.

12 Perracini MR, de Amorim JSC, Lima CA, et al. Impact of COVID-19 pandemic on Life-Space mobility of older adults living in Brazil: REMOBILIZE study. Front Public Health 2021;9:643640.

13 Rantanen T, Eronen J, Kauppinen M, et al. Life-Space mobility and active aging as factors underlying quality of life among older people before and during COVID-19 Lockdown in Finland-A longitudinal study. J Gerontol A Biol Sci Med Sci 2021;76:e60-7.

14 da Silva Castanheira K, Sharp M, Otto AR. The impact of pandemicrelated worry on cognitive functioning and risk-taking. PLoS One 2021;16:e0260061. 